\title{
Differenz
}

Revista internacional de estudios heideggerianos y sus derivas contemporáneas

AÑO 6 NÚMERO 5: JULIO DE 2019. e-ISSN: 2386-4877 - DOI: 10.12795/DIFFERENZ.2019.I05.08

[pp. 159-180]

Recibido: 15/04/2019

Aceptado: 25/06/2019

\section{El sentido fenomenológico del lenguaje poético en el segundo Heidegger}

\section{The phenomenological sense of poetic language in the second Heidegger}

\author{
César Alberto Pineda Saldaña \\ Benemérita Universidad Autónoma de Puebla
}

\section{Resumen:}

Este trabajo busca mostrar que el peculiar estilo poético que Heidegger emplea en sus obras ontohistóricas no es arbitrario ni responde a un mero afán de excentricidad. Al contrario, el filósofo vio en el lenguaje poético una manera de ser más fiel a su concepción de la fenomenología. La tesis propuesta consiste en que la fenomenología practicada en Sein und Zeit no alcanzó a dar cuenta del estado de arrojado (Geworfenheit) del Dasein ni de la esencia de la verdad entendida como rehúso. Para ello sería necesaria una fenomenología igualmente arrojada, acontecida, que encuentra su modelo rector en la poesía..

Palabras clave: Fenomenología; ontología; poesía; metodología; pensamiento ontohistórico. 


\section{Abstract:}

This paper seeks to show that the odd poetic style used by Heidegger within his ontohistorical works is not arbitrary, nor responds to a mere desire of eccentricity. Unlike this, the philosopher found poetic language as a way to be more faithful to his conception of phenomenology. The proposed thesis is that the phenomenology practiced in Sein und Zeit failed to account for the condition of thrown (Geworfenheit) of Dasein and for the essence of truth, understood as rejection. To do so, it would be necessary a phenomenology that is equally thrown, happened, which finds its guiding model in poetry.

Keywords: Phenomenology; Ontology; Poetry; Methodology; Ontohistorical Thought.

\section{Introducción.}

Cualquiera que, después de una lectura detenida de la obra heideggeriana de los años '20, haya pasado a los llamados tratados ontohistóricos, en particular a la que fue anunciada como la segunda gran obra, los Beiträge zur Philosophie, ha experimentado con toda probabilidad un profundo desconcierto. Tanto el estilo como el léxico de dichos escritos se resisten a una comprensión cabal. Esto ha traído dos consecuencias desafortunadas para una apropiación reflexiva y crítica del llamado segundo Heidegger: por un lado, mentes brillantes formadas en una línea fenomenológica, especialistas en la obra temprana, se han resistido a emprender una interpretación más detallada de la obra tardía, pues no ven en ella el mínimo asomo de rigor conceptual, de seriedad fenomenológica o metodológica; la otra consecuencia es que la misma ambigüedad aparente de la obra posterior al giro ha servido de refugio para interpretaciones poco rigurosas, que incluso imitan el estilo inspirado y poético del filósofo alemán. Desde tal ambigüedad casi cualquier cosa puede decirse a partir del pensamiento ontohistórico.

Inclusive voces cercanas a Heidegger expresaron su reserva con respecto al peculiar estilo de la obra tardía, a sus neologismos y arcaísmos -como escribir Seyn en lugar de Sein. Por ejemplo, Hannah Arendt comunicó a Karl Jaspers lo que a su modo de ver es un ridículo intento por separarse del mundo ordinario: "Esta vida en Todtnauberg, lanzando improperios contra la civilización y escribiendo Sein con y griega, en verdad es solamente la ratonera en la que se ha retirado" ${ }^{1}$. Asimismo, es conocida la dureza con la que Franco Volpi se refirió a los Aportes; en su introducción censurada a la traducción italiana de los

1 SAfRANSKI, R. Un maestro de Alemania. Martin Heidegger y su tiempo. México D.F., Tusquets, 2010. p. 433. 
Beiträge², Volpi describió a esta obra como la "bitácora de un naufragio", que si bien es imprescindible para la comprensión del pensamiento ontohistórico y el planteamiento del Ereignis, es después de todo “(...) una obra de transición, sustancialmente circunscrita al intento emprendido en los años de su redacción, pero luego dejado caer" ${ }^{3}$, y que "incluso sus geniales experimentaciones lingüísticas llegan a una implosión, y adquieren el aspecto de malabarismos, o más bien de vaniloquios" ${ }^{4}$.

¿Cómo posicionarse entonces ante las obras de este periodo? ¿Esos "malabarismos terminológicos" tienen algún sentido a partir del cual pueda entablarse un diálogo crítico y reflexivo con la filosofía heideggeriana de la Kehre? En lo que sigue se propone la hipótesis de que existen criterios fenomenológicos para dar cuenta del uso de un lenguaje quasi poético por parte de Heidegger; al comprender estos criterios, los peculiares términos de este periodo se tornan más legibles, y es reconocible el horizonte problemático al cual designan. Para ello será fundamental comprender el replanteamiento crítico que Heidegger hace de la fenomenología en los años '30, así como el papel protagónico que el lenguaje juega en este proceso.

\section{Consideraciones previas para plantear una unidad metodológica}

Una primera objeción puede saltar a la vista: ¿hay en verdad un proyecto metodológico, inclusive fenomenológico, en el contexto del pensamiento ontohistórico? La cuestión de la unidad temática en la obra heideggeriana es por sí mismo un problema sobre el que no hay consenso. Al respecto podemos encontrar tres perspectivas: una tesis de ruptura o diferencia entre un primer y un segundo Heidegger ${ }^{5}$; una de vuelta a lo primero, tomando la etapa fenomenológica de Ser y tiempo como un extravío $;$ y la tesis de una de relativa unidad ${ }^{7}$, de acuerdo con la cual Heidegger nunca pierde el interés central en la pregunta por el ser, lo que cambia es la concepción del fundamento del Dasein, que pasa de un planteamiento horizontal-trascendental a uno histórico. No es este el lugar para

2 Censurado en el sentido de que Hermann Heidegger, hijo del filósofo y administrador de su legado póstumo, se opuso a la publicación de dicho prólogo.

3 volPI, F. "Goodbye, Heidegger! Mi Introducción Censurada a los Beiträge zur Philosophie", en Actas del I Congreso Internacional de Fenomenología y Hermenéutica, Santiago de Chile, Universidad Andrés Bello, 2008, p. 61.

4 volPI, F. Martin Heidegger. Aportes a la filosofía. Madrid, Maia, 2010. p. 60.

5 Cfr. PöGgeleR, O. El camino del pensar de Martin Heidegger. Madrid, Alianza, 1993.

6 Cfr. GADAMER, H.-G. Los caminos de Heidegger. Barcelona, Herder, 2002

7 Cfr. HeRRMANn, F.-W. von. La segunda mitad de Ser y tiempo. Madrid, Trotta, 1997. 
analizar los pormenores de estas posturas, baste mencionar que este trabajo se basará en la tercera tesis, pues conforme aparecen cada vez más detalles de la obra póstuma se confirma que, independientemente de los giros y replanteamientos, la pregunta por el ser se mantuvo como leitmotiv del pensamiento Heideggeriano.

Pero la cuestión del método, si hay una relativa continuidad o ruptura con la fenomenología inicial, es menos clara todavía. Por razones que a continuación se expondrán, este artículo se inclinará por el proyecto de la unidad metodológica, más aún, de una unidad fenomenológica. Las estructuras existenciarias del Dasein, tema central de Sein und Zeit, conforman un primer ámbito que permite establecer un vínculo entre la tesis de la unidad temática y la de unidad metodológica. Entre quienes se han pronunciado por la unidad temática, Xolocotzi afirma que:

Las estructuras obtenidas del Dasein que se desplegaron en torno a la pregunta por su esencia no desaparecen al abandonar el fundamento ontológico-fundamental, el horizonte trascendental. Más bien son reinterpretadas a partir de un preguntar más originario por el fundamento de la esencia en la perspectiva histórica del ser ${ }^{8}$.

En un sentido similar, Vigo señala que la posterior concepción eventual-histórica de la verdad del ser no se caracteriza tanto por la inclusión de elementos externos o nuevos del todo, sino

(...) por la progresiva profundización de los momentos estructurales fundamentales avistados ya, de uno u otro modo, en el desarrollo de la analítica existenciaria, como ontología fundamental ${ }^{9}$.

La necesidad de nombrar estas estructuras de un modo propio, distinto al enfoque analítico y sistemático de los años '20, es lo que llevará a la apuesta por un lenguaje inspirado en la poesía. De este modo, antes de avanzar es necesario preguntar: ¿de qué modo el hecho de que los momentos estructurales del Dasein se mantengan, aunque replanteados, apoya la tesis de la unidad metodológica?

Para responder esta pregunta hay que precisar el sentido temático y metodológico de las estructuras existenciarias. En el marco de la fenomenología heideggeriana de los años '20 la vida fáctica del hombre es la instancia en la cual y como la cual es abierto el ser; siguiendo a Vigo, dicho nivel puede ser denominado "verdad de la existencia", la cual constituye el "objeto temático" de la fenomenología. El problema, a nivel "metodológico",

8 xolocotzl, A., Fundamento y abismo. México, BUAP-Porrúa, 2011. p. 67.

9 VIGo, A., Arqueología y aleteiología. Buenos Aires, Biblos, 2008. p. 182. 
es cómo acceder a dichas estructuras, cómo mostrarlas sin desfiguraciones en su "natural" suceder; de lograr esta mostración, se llega a una "verdad fenomenológica", cuyo sentido es siempre "llegar a emparejarse" con la verdad de la existencia.

Las estructuras existenciarias son tan singulares, y por ello difíciles de avistar, porque deben dar cuenta de un ente que en cierto modo "está siendo", que existe como pro-yecto, a diferencia de cualquier otro ente "acabado y a la mano". Ser y tiempo es el progresivo intento por ir ganando claridad fenomenológica sobre dichas estructuras. Pero hay algo que la fenomenología de corte "existencial, individual y singularizante" no llega a captar: el "estado de yecto o arrojado" (Geworfenheit) del Dasein que acontece al modo de la historicidad, pues como señala von Herrmann:

La perspectiva de la Historia del Ser es resultado de una mutación inmanente de la perspectiva de la Ontología Fundamental en cuanto trascendental y horizontal. Esta mutación inmanente se pone en movimiento en la experiencia fenomenológica, al no agotarse el carácter de yecto de Apertura del ser ${ }^{10}$.

A esto debemos añadir el replanteamiento de la idea de verdad. Entre 1929 y 1930, en textos como Vom Wesen der Wahrheit, Heidegger comenzará a enfatizar el elemento "sustractivo" o "rehusante" como auténtica esencia del des-ocultamiento. Al respecto, Alejandro Vigo ha señalado que

(...) la noción de "sustracción", extrapolada hacia un plano de consideración diferente, juega un papel clave en la transición hacia la concepción eventualhistórica de la verdad del ser, característica del pensamiento de la Kehre ${ }^{11}$.

Este es un cambio temático fundamental, ya que en Ser y tiempo aún se consideraba que el momento más esencial de la verdad de la existencia, y por ende de la verdad fenomenológica, era el de la "claridad"; lo que "encubre", la no-verdad, es algo que debe ser superado y aclarado en un adecuado acceso fenomenológico. Esta interpretación se confirma con la reciente aparición del tomo 82 de la Gesamtausgabe, en el cual, hacia 1936, Heidegger emprende una revisión crítica de Ser y tiempo: "En Sein und Zeit la noverdad (Un-Wahrheit) es tomada de modo aún más negativo -el no-todavía, lo que en realidad tendría que ser remontado"12. En cambio, desde la nueva perspectiva "/a no-

10 HERRMANN, F.-W. von. op. cit. p. 91.

11 VIGO, A. op. cit. p. 168.

12 HEIDEGGER, M. GA 82. Zu eigenen Veröffentlichungen. Fráncfort del Meno, Vittorio Klostermann, 2018. p. 112. (Trad. propia). Con el objetivo de no saturar las notas a pie de página, para la bibliografía primaria y secundaria citada que no cuente a la fecha con una traducción al español, ofrecemos 
verdad es la auténtica esencia de la verdad, en tanto que es puesta metafísicamente como esencia del Da-sein"13.

Así pues, al considerar que la esencia de la verdad es un "rehusante ocultamiento", la existencia cotidiana del hombre, que se mueve en la relativa claridad y disponibilidad, ya no puede ser el momento fundamental del desocultamiento, y en correspondencia con esto, la verdad fenomenológica, junto con su lenguaje, deberá ahora captar el esencial ocultamiento. Esto traerá nuevos problemas y la necesidad de replanteamientos, pero respondiendo siempre al mismo objetivo: una "simultaneidad" ente verdad fenomenológica y verdad del Dasein. ¿Es posible hablar de una fenomenología en la obra de los años 30? Otra intérprete que apuesta por dicha posibilidad es Leticia Basso:

Cuando Heidegger inaugura el estilo del Seynsdenken, particularmente en el proyecto en curso de los Beiträge, pretende transformar la búsqueda fenomenológica iniciada con la analítica existencial, aunque manteniendo algunos rasgos de la estructura del Dasein que para él siguen siendo centrales a la hora de efectuar der kehriger Bezug des Seyns ${ }^{14}$.

La palabra "estilo" que emplea Basso no es gratuita; el peculiar lenguaje y forma de escritura que hallamos en los textos de la época de los Aportes no es fortuito y, como se verá, responde a la idea de fenomenología que Heidegger sigue en esos años. Es momento, pues, de mostrar si hay una fenomenología en el pensamiento ontohistórico. Hay tres perspectivas sobre el tema: en primer término, quienes consideran que no solo falta una postura explícita sobre el método, sino que dicho pensar es esencialmente "ametódico", postura iniciada por Gethmann"15; en segundo lugar quienes observan un cambio de método, que ya no es fenomenológico, lectura comenzada por Pöggeler ${ }^{16}$; y finalmente quienes, como von Herrmann ${ }^{17}$, afirman que el método fenomenológico se mantiene.

una traducción propia y omitimos el fragmento en su idioma original. El interesado en cotejar los términos alemanes puede remitirse a las páginas citadas.

$13 \mathrm{lb}$.

14 BASSO, L. La unidad de la diferencia. Buenos Aires, Biblos, 2017. p. 241.

15 Cfr. Gethmann, C. Verstehen und Auslegung. Das Methodenproblem in der Philosophie Martin Heideggers. Bonn, Bouvier, 1974.

16 Cfr. PÖGGELER, O., op. cit.

17 Cfr. Herrmann, F.-W. von. Weg und Methode. Zur hermeneutischen Phänomenologie des seinsgeschichtlichen Denkens. Fráncfort del Meno, Vittorio Klostermann, 1990. 
Probablemente la objeción más significativa para esta última interpretación la constituye el hecho de que no encontremos una mención explícita de Heidegger ni a la fenomenología, que pareciera simplemente haber quedado atrás, ni a ninguna aclaración metodológica en general. Von Herrmann no ve ningún problema en esta circunstancia, basta con enfatizar el carácter "hermenéutico" de la fenomenología a partir de la Kehre. En cambio, aunque dentro de la misma interpretación de la unidad metodológica, para Oliver Cosmus no es secundario que la palabra "fenomenología" no aparezca más, sino que revela el trasfondo de la idea de fenomenología que Heidegger desplegará a partir de los años '30; se trataría de una "fenomenología anónima”. Cosmus enuncia su tesis del siguiente modo:

\section{La unidad del camino en el pensar de Heidegger radica en el método} fenomenológico que lo conduce. La fuerza conductora en la marcha del pensamiento heideggeriano de Ser y tiempo a los Aportes y a los Hitos de su pensamiento tardío es, como deberá mostrarse, la genuina referencia fenomenológica al fenómeno del $\operatorname{ser}^{18}$.

La posterior fenomenología sería, entonces, "anónima", sin la sistematicidad ni desarrollo dialéctico que se observa en Sein und Zeit; aunque el profesor de Friburgo rechace el título "fenomenología", la máxima "a las cosas mismas" seguirá rigiendo su trabajo incluso con mayor intensidad que antes ${ }^{19}$. En Zu eigenen Veröffentlichungen Heidegger escribe en su relectura de Ser y tiempo: "Fenomenología como título para ese camino a la cosa -a saber, el de la filosofía, y eso quiere decir, a la meditación sobre sus preguntas esenciales y necesarias" 20.

18 cosmus, O. Anonyme Phänomenologie. Die Einheit von Heideggers Denkweg. Würzburg: Königshausen \& Neuman, 2001. p. 13.

19 Como es sabido, desde el §7 de Sein und Zeit Heidegger reforzó y difundió la idea de que el lema "a las cosas mismas" es una indicación sobre el esencial propósito de la fenomenología. Estas cosas mismas son los fenómenos en su comparecencia o desocultamiento. La interpretación de Cosmus se apoya en la hipótesis de que Heidegger agudizará su fidelidad a dicho lema. No obstante, vale la pena recordar que dentro de la fenomenología hay hasta la fecha un debate sobre el sentido de la máxima fenomenológica. Antonio Zirión sostiene que el lema es solo una indicación formal que atañe a toda actividad que pretenda ser científica; por su parte, Ángel Xolocotzi afirma que, si bien de inicio es un postulado formal, puede y de hecho ha recibido un despliegue concreto como caracterización de la fenomenología misma. Cfr., sobre este debate, el libro coescrito por ambos filósofos, iA las cosas mismas! Dos ideas sobre la fenomenología (México, Porrúa, 2018). En todo caso, lo que parece fuera de duda es que Heidegger entiende el famoso lema como guía para la labor más propia de la fenomenología.

20 HEIDEGGER, M. GA 82. cit. p. 145. (Trad. propia). 
En una línea de interpretación semejante a la de Cosmus, Basso habla de una fenomenología de lo inaparente (Phänomenologie des Unscheinbaren), desarrollada inclusive en los últimos trabajos del filósofo (como en los seminarios de Zollikon, Le Thor y Zähringen). Igualmente, en el protocolo de un seminario sobre Tiempo y ser, Heidegger reconoce explícitamente que el proceder de dicha conferencia, leída en 1962, fue fenomenológico:

Este proceder puede ser caracterizado como fenomenología, en la medida en que por fenomenológico no se entienda ningún tipo particular y dirección de la filosofía, sino algo que campea en toda filosofía ${ }^{21}$.

De acuerdo con Basso, este proyecto fenomenológico tardío buscaría volver a la diferencia ontológica

(...) con una postura más radical que lo orienta al fundamento abismal de la donación. Esta nueva versión de la fenomenología termina de despejar cualquier atisbo de cientificidad del método ${ }^{22}$.

Veamos ahora con más detalle cómo se sostiene la idea de una fenomenología "anónima" o "inaparente".

Cosmus observa que en el desarrollo de la analítica existenciaria de 1927 el método fenomenológico solo está presupuesto y co-tematizado, pero siempre faltó una exposición expresa y detallada del proceder metodológico; el §7 de Sein und Zeit no hace sino ofrecer un primer y breve esbozo. Una razón para ello es que la obra del '27 está organizada de cara a sus resultados, no tanto al esclarecimiento del proceder metodológico; es por esto que el tratado,

(...) después de un (provisional) esclarecimiento de la pregunta metodológica, ha de ponerse por completo al servicio de la problemática de la cosa misma.

En este retirarse detrás de la pregunta por la cosa radica el primer paso de la fenomenología hacia su propio anonimato ${ }^{23}$.

El método pierde protagonismo, debe "sustraerse" para dejar que se muestre por sí mismo el asunto temático por pensar. En favor de este anonimato hablan las palabras de Heidegger en Mi camino en la fenomenología, texto de 1963:

21 HeIDEgGeR, M. Tiempo y ser. Madrid, Tecnos, 2011. p. 78.

22 BASSO, L. op. cit. p. 181.

23 cosmus, O. op. cit. p. 49. 
(...) la fenomenología no es dirección alguna, sino que es la posibilidad del pensar que, llegados los tiempos, reaparece de nuevo, variada, y que solo por ello es la permanente posibilidad del pensar, para corresponder al requerimiento de aquello que hay que pensar. Cuando la fenomenología viene así experimentada y conservada, puede entonces desaparecer como rótulo en favor de la Cosa del pensar, cuya revelabilidad sigue siendo un misterio ${ }^{24}$.

Si en Ser y tiempo solo fue esbozado un proyecto preliminar de fenomenología, el desarrollo expreso y más acabado de una idea de la fenomenología estaría prometido en la tercera sección de Los problemas fundamentales de la fenomenología, titulada "La forma científica de tratar estos problemas y la idea de la fenomenología" 25 , pero esta tampoco llegó a realizarse. Dicha idea de la fenomenología es algo que se formaría en el transcurso del propio desarrollo temático, es decir, aún no estaba hecha y acabada, sino en proceso de ser fundamentada. Este puede ser el sentido de la conocida frase del $\S 7$ de Ser y tiempo, en donde asevera con respecto a la fenomenología que "por encima de la realidad está la posibilidad. La comprensión de la fenomenología consiste únicamente en aprehenderla como posibilidad"26.

Preguntemos, pues, ¿̇por qué debería ser "anónima" o "inaparente" la fenomenología en el paso al pensamiento ontohistórico? El replanteamiento fenomenológico corresponde a un replanteamiento temático, el cual hemos tenido oportunidad de observar en sus rasgos generales: lo que cambia en el paso de la analítica existenciaria al pensamiento ontohistórico es el énfasis en el momento "sustractivo" y "acontecedor" de la esencia de la verdad. A partir de esta modificación "temática", el método debe replantearse en consecuencia, debe corresponder a esa sustracción. Cosmus señala que en este paso se pierde el nombre "fenomenología" en favor del sobrio "pensar" (Denken). Un proyecto metodológico, que sea explícito y planificado no permitiría "acceder" ni al estado de arrojado del Dasein (Geworfenheit) ni al rehúso del des-ocultamiento; una fenomenología planificada y controlada es una última reminiscencia del sujeto moderno. El método deberá, entonces, "sustraerse" para estar en sin-tonía con el esencial rehúso de la verdad. Si hay un método de acceso este debe ser "acontecedor" como la verdad misma del ser, una "fenomenología que acontece". ¿Cómo sería esto posible?

24 HEIDEGGER, M. Tiempo y ser. cit. p. 124.

25 Cfr. HEIDEGGER, M. Los problemas fundamentales de la fenomenología. Madrid, Trotta, 2000. p. 25 y ss.

26 HeIDEGGER, M. Ser y tiempo. Madrid, Trotta, 2012. p. 58. 


\section{El papel metodológico del lenguaje poético en una fenomenología inaparente}

Surge aquí el problema del lenguaje y de un adecuado nombrar, cuestión presente desde los inicios de la fenomenología. Husserl se percató desde un comienzo del problema de la modificación de los actos en su tránsito de una actitud natural a la actitud reflexiva o fenomenológica, y aunado a esto, también el problema de la selección de los términos adecuados para nombrar y distinguir entre las vivencias naturales y su análisis reflexivo una vez que ha pasado por el crisol de la fenomenología, es decir, de la actitud "reflexiva":

Es absolutamente imposible describir los actos de mención, sin recurrir en la expresión a las cosas mentadas. Y icuán fácil es entonces olvidar que esa "objetividad», incluida en la descripción y que necesariamente ha de ser tenida en cuenta en casi todas las descripciones fenomenológicas, ha recibido una modificación de sentido, por la cual pertenece ella misma a la esfera fenomenológica! ${ }^{77}$

Con respecto a las vivencias de la actitud natural que buscan nombrar, los términos elegidos por la fenomenología no deben ser tan lejanos y extraños que aquellas vivencias queden irreconocibles, pero tampoco tan próximos a ellas que resulten indiscernibles. Por tal motivo, Husserl evita proponer neologismos y términos irreconocibles para la tradición. Un ejemplo de esto es el mismo término de "acto"; el filósofo moravo observa que dicha palabra conlleva una dificultad, pues alude a una idea fuerte de "actividad", algo que queda excluido de su tratamiento de los actos -es perfectamente concebible que haya actos espontáneos en la conciencia, actos "pasivos". Ante ello, parecería recomendable buscar otro término o proponer un neologismo; pero es una dificultad que se debe sostener a cambio de no resultar ilegible o ininteligible. Husserl considera entonces:

Si no queremos introducir términos técnicos enteramente nuevos, extraños

a todo vivo sentido del lenguaje y a toda tradición histórica, casi nunca podremos evitar dificultades de la índole que acabamos de exponer ${ }^{28}$.

Husserl transige, pues, con la tradición; aunque el contenido de sus conceptos sea en ocasiones enteramente distinto, a veces contradictorio, al sentido de la tradición filosófica, decide mantener el mismo término para poder dialogar con ella. Esto sucede con términos como "yo", "conciencia", "trascendencia," que sin duda tienen una carga semántica muy diferente a la que habían tenido en la filosofía moderna. El mismo criterio se mantiene

27 HUSSERL, E. Investigaciones lógicas I. Madrid, Alianza, 1999. p. 222.

28 Ib. p. 499. 
en Ideas, donde el filósofo considera que “(...) no es admisible elegir expresiones técnicas que se salgan totalmente del marco del lenguaje histórico de la filosofía" 29.

Heidegger, con una estrategia nominativa opuesta, consideraría que la única manera de ser fiel a la vivencia natural, a la "cosa misma", tal como ella es dada, es romper con los términos de la tradición que desfiguran con su reflexividad teorética el "natural suceder" de los actos. En realidad, el objetivo para el discípulo de Husserl es el mismo: encontrar términos cercanos a la vida fáctica, pero que no se confundan con el habla cotidiana; términos que permitan un discernimiento temático de la esfera "atemática", pero no tan extraños que esta última ya no se muestre en ellos. Un balance adecuado es difícil de lograr. Ya que Heidegger busca un acceso a la vivencia fáctica, los términos de la fenomenología deberán tener el mismo carácter "vivencial". ¿Qué expresión "temática" puede y debe nombrar la vivencia "atemática"? Con respecto a esta cuestión, Xolocotzi explica lo siguiente:

[...] a este tematizar expreso también pertenece otro modo de entender la expresión y la formación conceptual. En concordancia con esta reflexión Heidegger dirá desde 1919 que la expresión que corresponde a la ciencia originaria debe entenderse como un carácter del aprehender concreto de la vida misma ${ }^{30}$.

El término elegido debe, pues, permitir una re-sonancia de la vida fáctica, de ahí la elección de vocablos "poco científicos" que inevitablemente suenen a algo mundano, pero al mismo tiempo "inusuales", como son Verfallen, Uneigentlichkeit, Schuld, Gewissen, Sorge. Dado que para Heidegger la tradición filosófica ha enfatizado la perspectiva teóricoreflexiva, no debe sorprender que ella elija también términos con énfasis teorético; en esta línea se encontraría Husser ${ }^{31}$. Xolocotzi añade:

29 HUSSERL, E. Ideas relativas a una fenomenología pura y una filosofía fenomenológica. Libro primero: Introducción general a la fenomenología pura [Ideas I]. México D.F. UNAM/FCE. 2013, p. 83.

30 xolocotzl, A. Fenomenología de la vida fáctica. México D. F, Plaza y Valdés, 2004. p. 53.

31 Es pertinente hacer una aclaración sobre esto: durante cierto tiempo se volvió usual la división esquemática entre un Heidegger hermenéutico y un Husserl teorético. Como en cualquier fórmula establecida, hay algo de cierto y mucha generalización excesiva. Este esquema llevó a la idea de que Husserl no se habría interesado por el mundo atemático y cotidiano sino hasta su obra tardía, especialmente en Die Krisis der europäischen Wissenschaften, de 1938. Heidegger contribuyó a diseminar esta idea. No obstante, hoy es conocido, al tener acceso a las lecciones tempranas de ambos autores, que Husserl ya bosquejaba algunas ideas relativas a la temática del Lebenswelt al menos desde sus lecciones en Friburgo de 1919, época en la que su discípulo Heidegger se desempeñaba como Privatdozent y trabajaba en torno al concepto de Umwelt. Así 
(...) los conceptos heredados de la tradición que buscan tematizar la vida en términos filosófico-originarios, por ejemplo, sujeto, conciencia, yo, persona, y otros, están ya cargados teoréticamente. Por ello deben ser reconstruidos críticamente $^{32}$.

De aquí resultarán términos y abordajes como los de Dasein, Selbstheit, Jemeinigkeit. La primera apuesta nominativa de Heidegger la constituirán, desde sus tempranas lecciones en Friburgo, las "indicaciónes formales", antecedentes directos de los existenciarios de Ser y tiempo, términos que tienen un pie puesto en la facticidad y otro en la conceptualidad. De este modo, “(...) la indicación formal debe ser vista como la conceptuación ejecutante que puede conducir a un despliegue temático del fenómeno vida" ${ }^{33}$. Resulta clave la idea de una "concepción ejecutante"; el término elegido debe ser él mismo una vivencia acontecida, en un lenguaje vivo.

Ahora bien, el propósito de que el lenguaje filosófico esté en con-sonancia con el ámbito del "suceder" fáctico no cambiará con la Kehre; más bien, esta última es una consecuencia de aquel propósito. Pero si lo que se busca es esa "simultaneidad consonante" entre fenomenología y facticidad, cabe la pregunta de si realmente es viable un acceso teórico, conceptual, al nivel preteorético. ¿No tendría que perder la fenomenología su carácter teorético y científico para corresponder a la vida preteorética? ¿No tendría que "diluirse" la fenomenología como método reflexivo en el anónimo y contingente suceder de la historia y la facticidad? La fenomenología tendría que ser anónima e inaparente, justo como lo es el acontecer. Pero con ello viene aparejada, como supo ver Husserl en su lectura crítica de Ser y tiempo, la posibilidad de que la fenomenología pierda su carácter científico.

Igualmente es preciso considerar el cambio temático al que hemos aludido, a saber, el posterior énfasis en el momento "rehusante" de la verdad, y el fundamento históricoacontecedor del Dasein. En consonancia con esto, la fenomenología deberá cambiar de matices. Lo sustractivo de la verdad se da como historia del ser, a partir de lo cual observa Cosmus: "En la medida en que ya no se puede preguntar por un principio del acontecer

pues, temáticamente ambos filósofos se interesaron por el "mundo de vida atemático"; pero resulta difícil y en todo caso superfluo quién abordó primero el problema. Lo cierto es que ante el mismo "ámbito atemático" Heidegger buscó un acceso igualmente vivencial y atemático, a pesar de todo "tematizante", y Husserl buscó asegurar un acceso temático, reflexivo y decididamente científico. El punto de quiebre no es el tema o el objeto, sino el modo de acceso.

32 xolocotzı, A. Fenomenología de la vida fáctica. cit. p. 171.

33 Ib. p. 123. 
del ser (Seinsgeschehens), este último se muestra como un proceso 'anónimo'”34. Un acceso fenomenológico a dicho proceso anónimo debe ser igualmente anónimo. ¿Cómo podría lograrse esto? Existe además otra dificultad; cualquier concepto para nombrar tal acontecer corre el peligro de objetivarlo, incluso el término Ereignis; de ahí que los esfuerzos de la fenomenología estén siempre, necesariamente, "a la zaga del acontecer". Esta fenomenología anónima es entonces, inevitablemente junto con los términos que elige, "provisional", algo que tal vez sabía Heidegger desde Ser y tiempo. Pero su provisionalidad no es un accidente o defecto, sino algo necesario en su proceder. Basso indica que "con lo inaparente (das Unscheinbare) Heidegger mienta la falta de una determinación acabada del sentido del ser debido a su condición inefable" ${ }^{35}$.

Tampoco debería sorprendernos, entonces, que el profesor de Friburgo no haya presentado jamás una formulación última y definitiva de la fenomenología, porque tal cosa no la puede haber. De esta manera, en el pensamiento de la Kehre "el modo de acceso preteorético a la vida fáctica o Dasein, al que nos hemos referido, es un modo de acceso que no será abandonado" ${ }^{36}$, inclusive se radicalizará. Pero al preguntar por el fundamento rehusante de la verdad del ser, las dificultades metodológicas no harán más que acentuarse: ¿cómo acceder a lo que es por definición "inaccesible" (el "rehúso")? Ello como tal se sustrae, pero deja huellas, señas, pistas (Winke, Spuren, Hinweise), una idea crucial para la "fenomenología inaparente" de los años 30.

Hacia 1939 Heidegger recuerda que el ser no es un ente, pero

(...) este no-ente es frente a todo ente el rehúso en el que el ser (Seyn) se retira a su más propia esencia y hace señas de sí como el origen (Ur-sprung), donde el no tiene su procedencia ${ }^{37}$.

La expresión en el original alemán es “(...) als den Ur-sprung anwinkt" ${ }^{38}$. El ser se retira, pero su retirada es ella misma una seña esencial. En el curso inmediatamente anterior a la redacción de los Beiträge, Preguntas fundamentales de la filosofía, el filósofo habla de un discernimiento esencial sobre el ocultarse del ser; el ente no solamente está delimitado por algo oculto, el ser, sino que este mismo se oculta:

34 cosmus, O., op. cit. p. 101.

35 BASSO, L. op. cit., p. 181.

36 xolocotzı, A. Fenomenología de la vida fáctica. cit. p. 203.

37 heidegger, M. Meditación. Buenos Aires: Biblos, 2006. p. 62 (Cursiva nuestra).

38 HeIDEGgeR, M. GA 66. Besinnung. Fráncfort del Meno: Vittorio Klostermann, 1997. p. 58. 
Su ocultarse es por ello de una especie originariamente propia. Se muestra $\mathrm{y}$ al mismo tiempo se retrae. Este denegarse vacilante es aquello que propiamente está despejado en el claro (Lichtung) ${ }^{39}$.

La esencia rehusante de la verdad del ser no es ni mera mostración ni simple ocultamiento absoluto, es como el tintineo de la estrella más lejana, a penas visible. Por ello agrega que "(...) tampoco este ocultarse es un mero estar ausente, sino denegación vacilante" ${ }^{\prime 40}$. En tal vacilar se abre una seña. A partir de esto, el lenguaje de la fenomenología inaparente debe tener el mismo el carácter de una seña, debe brillar también para luego vacilar y finalmente sustraerse. Si tenemos esto en cuenta, no es para nada superfluo el título que el filósofo alemán elige para los primeros Cuadernos negros, Überlegungen und Winke. Las señas (Winke) serán el tercer gran intento de una estrategia nominativa por parte de Heidegger, después de las indicaciones formales y los existenciarios.

La seña tendrá un radical carácter de "simultaneidad": es al mismo tiempo seña del ser y del lenguaje pensante que está en sin-tonía con aquel. Por ejemplo, el término Machenschaft, que como ya sabemos es un primer intento por nombrar la esencia de la técnica, es una seña de este tipo:

La seña (Winke) más calma del ser (Seyn), a través de la cual éste hace señas de sí, es la indecisión de esa resolución en la figura del abandono del ser al ente, que se afirma como maquinación (Machenschaft) en supremacía ${ }^{41}$.

Habría que leer todas las expresiones de esta época -Füge, Zerklüftung, Geviert, Verhaltenheit, Anklang, entre muchas otras- como "señas fenomenológicas", y no como meras extravagancias de un autor obsesionado con lo heterodoxo y oscuro. Tales "señas" no son ya indicadores formales o conceptuales que traten "sobre" algún fenómeno o acontecer, sino que buscan "insertarse" en ese mismo acontecer, formar parte de él como algo intrínseco, no externo. Por ejemplo, con respecto a la noción misma de Kehre, lain Thompson señala que en los primeros Cuadernos negros el filósofo alemán no habla "sobre" la Kehre sino "desde" ella, y más aún, guarda silencio sobre ese giro:

39 heidegger, M. Preguntas fundamentales de la filosofía. "Problemas" selectos de "lógica". Granada, Comares, 2008. p. 192.

40 lb. p. 193.

41 HEIDEGGER, M. Meditación. cit. p. 290. 
Heidegger toma un voto de silencio sobre este giro en su trabajo, con la finalidad de dejar que su trabajo mismo anuncie más efectivamente ese giro, primero y sobre todo a través de llevarlo al acto ${ }^{42}$.

Por otra parte, si en la etapa previa de una "fenomenología existencial" los fenómenos de acceso eran vivencias singularizantes como la angustia, desde la perspectiva ontohistórica los fenómenos de acceso al ser, nombrados como Winke, no serán ya vivencias personales sino, por sí mismos, "eventos históricos", como la Machenschaft o el Ereignis. Es a través de estas señas, o más precisamente, "como tales señas" -pues ya no son solamente un "medio"- que es posible establecer una relación pensante con el ser.

Líneas arriba se mostró que el acceso metódico debe cambiar para dar cuenta del anónimo acontecer. Aquí se encuentra la importancia del lenguaje como "zona" donde puede suscitarse el acontecer del pensar: "Ella debe corresponder a la afluencia histórica, es decir: ser fundada por el poeta"43, a la manera de la poesía. Por ello el peculiar lenguaje, que podría calificarse como "criptopoético", de textos como los Beiträge; debe ser un lenguaje "acontecido" como lo es la poesía. Se trata de un lenguaje que "adviene" al hombre, no es su creación consciente y voluntaria, como todavía son los "indicadores formales". En el apéndice de la conferencia Fenomenología y teología, escrito en 1964 (la conferencia se dictó en 1927), el filósofo escribe que

(...) el secreto del lenguaje, en el que se tiene que concentrar toda la reflexión, sigue siendo el fenómeno más cuestionable y más digno de ser pensado, sobre todo cuando se alcanza a comprender que el lenguaje no es una obra del hombre: el habla habla ${ }^{44}$.

Un poco más adelante añade: “(...) el pensar es siempre un dejarse decir por parte de lo que se muestra y por lo tanto un corresponder (decir) frente a lo que se muestra" ${ }^{45}$.

Cabe destacar que Heidegger siempre se refiere a dicho lenguaje como "provisional", pues nunca hay una correspondencia inequívoca al rehúso del ser ${ }^{46}$. Recordemos algunas palabras del epígrafe que el autor coloca al inicio de los Beiträge: "lo contenido en larga

42 THOMson, I. "Heidegger's Nazism in the Light of his early Black Notebooks: A view from America", en DENKER, A. y ZABOROWSKI, H. (eds.), Heidegger-Jahrbuch 11. Zur Hermeneutik der "Schwarzen hefte". Freiburg/Múnich, Karl Alber, 2017, p. 194.

43 cosmus, O., op. cit., p. 101.

44 HeIDEgGer, M. Hitos. Madrid, Alianza, 2007. p. 69.

$45 \mathrm{lb}$. p. 71.

46 En consonancia con esto, el filósofo escribe en 1941: "El ser (Seyn) sólo puede ser nombrado por sí mismo, él mismo es el dicho. Y lo difícil para el hombre muy hablador es encontrar en una palabra 
vacilación, indicadoramente retenido". O las líneas, al inicio de las Reflexiones X, que sirven como presentación para el conjunto de los Cuadernos negros: "inaparentes puestos de avanzadilla y de retaguardia en el conjunto del intento de una meditación, todavía indecible". Richard Polt ha subrayado recientemente que los Cuadernos negros, al ser diarios de pensamiento, no responden a un plan de escritura previo; todo apunta, más bien, a que su redacción se daba de cierta manera espontánea, como un "acontecer", ya que en la escritura de un diario rara vez se tiene una idea planificada de la forma final del escrito, más bien, "la idea toma forma conforme es escrita y el autor puede ser sorprendido por una pregunta, paradoja u objeción que surge en el curso de la escritura" ${ }^{47}$. El hecho de que no haya borradores previos, como señala el editor de estos volúmenes, Peter Trawny, puede ser inicio de que no hubo intentos de pulir, corregir o reflexionar los pensamientos que simplemente surgían y que por tanto, tal vez, permanecieron en su crudeza e inmediatez. Desde este enfoque Thomson también observa que las modificaciones más notables en los Cuadernos negros incluyen una visión en la que Heidegger apunta

(...) a la naturaleza polisémica del ser y su consecuente desarrollo de un estilo poético necesario para hacer justicia a las riquezas fenomenológicas aparentemente inagotables del ser. ${ }^{48}$

No solo el estilo, sino la semántica y la estructura misma de los escritos de esta época resultan de tal modo "criptopoéticos", pues el lenguaje del pensar busca acontecer "como" lo hace la poesía. Este último "como" es relevante, pues tampoco se trata de poesía sin más, sino que tal lenguaje todavía guarda un propósito metodológico. De acuerdo con esta pauta, textos como los Aportes dejan de ser "descriptivos", como todavía puede entenderse Ser y tiempo, que buscaba precisamente "describir" las estructuras existenciarias del Dasein; con la "fenomenología inaparente" de los años 30, el decir ya no está frente a un objeto por decir (las estructuras del Dasein), sino que él debe "ser el acontecer" del pensar histórico. Por eso en su revisión crítica de Ser y tiempo el profesor de Friburgo señala: "La pregunta por el ser del Dasein no es la búsqueda de una adecuada descripción del Dasein (como si "estuviera ya a la mano"), sino 'efectuación del ser (Seyn) del ahi'"'49.

En este sentido, observa Daniela Vallega-Neu,

esencial lo suficiente para el encarecimiento en lo esencial", HEIDEGGER, M. Sobre el comienzo. Buenos Aires, Biblos, 2007. pp. 34-35.

47 Polt, R. "The Black Notebooks as Thought Journals", en DENKER, A. y ZABOROWSKY, H. op. cit. p. 47. 48 THOMSON, I. op. cit. p. 185.

49 HEIDEGGER, M. GA 82. cit. p. 51 (Trad. propia). 
Para Heidegger la tarea central de los Beiträge zur Philosophie es la fundación del Da-sein, esto es, un decir pensante, en el cual el Da-sein gana permanencia y la posibilidad de otro comienzo de la historia del ser se mantiene abierta ${ }^{50}$.

De acuerdo con este enfoque, la fenomenología debe borrarse como tal, en tanto medio o método, para ser, como pensar y decir, el "acontecer" mismo. La fenomenología descriptiva de los años '20 aún implicaba, en tanto descripción, una distancia, una diferencia con respecto al objeto temático; en cambio, la "fenomenología inaparente", que acontece en tanto seña (Winke), busca la mayor cercanía posible con el asunto del pensar, al grado que ella misma sea la fundación del asunto del pensar.

La fenomenología de Ser y tiempo aún se insertaba en un proyecto trascendental, es decir, buscaba "condiciones de posibilidad", y como tales eran pensadas las estructuras ontológicas del Dasein. En cambio, escribe el pensador en los Cuadernos negros: "Con la tarea de la fundamentación del Dasein con el pensamiento y la poesía se ha superado la pregunta por la posibilidad"51. El pensar poetizante busca ser él mismo el acceso fenomenológico, como quizá lo fue en los inicios del pensar occidental, con Anaximandro, Heráclito y Parménides: "La poesía no es solo comunicación de posibilidades existenciales, sino fundación del ser, nombrar originario de los creadores -salto originario del $D a$ $\operatorname{sein"}{ }^{52}$. Encontramos por lo tanto una "simultaneidad" entre "palabra, pensamiento y verdad del ser", en dicha simultaneidad se fundamenta la posibilidad de "ser apropiado (ereignet) por y para el ser"; la fenomenología inaparente es, pues, posibilidad de fundación del Ereignis, y el lenguaje será pensado en este punto como "experiencia apropiante". Al respecto observa Vallega-Neu: “(...) la experiencia de la que habla aquí Heidegger está relacionada con varias formas de actividad creativa. Tomemos como ejemplo la escritura creativa o la poesía" ${ }^{53}$.

En este sentido pueden leerse las siguientes líneas de las Reflexiones VI:

Para la filosofía -y máxime para aquella que supera toda metafísica como algo que queda en primer plano-, la palabra y su configuración son el

50 VAlLEGA-NEU, D. “Die Schwarzen Hefte und Heideggers seynsgeschichtliche Abhandlungen (19361942)“, en DENKER, A. y ZABOROWSKY, H. op. cit. p. 106.

51 HEIDEGGER, M. Cuadernos negros (1931-1938). Madrid, Trotta, 2015. p. 178.

52 HEIDEGGER, M. GA 82. cit. p. 89.

53 VAlLegA-NeU, D. op. cit., p. 105. 
acontecimiento del ser (Seyn) mismo: el ser como acontecimiento que nos hace apropiados a ella" ${ }^{54}$.

Se trata de un "decir acontecimental" en el que se borra la separación que se daba con el "acceso reflexivo"; la palabra pensante no es susceptible de planificación metódica, sino que se da. Como acceso al acontecer del ser, la poesía y el pensamiento

(...) entran en una relación transformada esencialmente, la cual no se puede calcular. Cuándo y cómo ambos se hacen patentes como Da-sein dentro de lo ente que se transforma, sin ser y sin "repercutir" públicamente, eso es algo que no sabe nadie ${ }^{55}$.

Al "surgir" y transformarse continuamente, los términos de esta época no son consistentes ni sistemáticos, muchos de ellos aparecen para ser abandonados después de algunos tanteos; no debe extrañar que la mirada pública encuentre este lenguaje extravagante, oscuro e incluso "sin sentido".

En realidad, reflexiona Heidegger en el segundo volumen de los Cuadernos negros, todavía no sabemos nada de la incardinación esencial del ser y del lenguaje (...) el lenguaje nunca es primero "expresión" ni "medio de formulación" de un pensamiento, sino el encaje original (ursprüngliche Fügung) del pensar y lo pensado mismos ${ }^{56}$.

Con esto se radicaliza una idea temprana de Heidegger, que el lenguaje no es un mero instrumento; se trata más bien del ámbito en el cual el ser "se apropia" del hombre, en la esencia más íntima del lenguaje "se da" el ser:

Los grados de la irradiación de la esencia de la palabra que van ascendiendo a partir del uso léxico inmediato son estos: la palabra designa, la palabra significa, la palabra dice, la palabra es ${ }^{57}$.

Entre más se avanza en estos grados de irradiación, hay una mayor equivocidad esencial del lenguaje, misma que solo dominan poetas y pensadores. De acuerdo con tal equivocidad esencial hay que leer los Winke de esta época. Sólo un lenguaje asumido como medio e instrumento puede ser inequívoco.

54 HEIDEGGER, M. Cuadernos negros (1931-1938). cit. p. 406. (Trad. modificada). 55 HEIDEGGER, M. Cuadernos negros (1938-1939). Madrid, Trotta, 2017. p. 129. 56 Ib. p. 85.

57 Ob. p. 143. 


\section{A manera de conclusión}

Así pues, "la palabra acontece como ser y como pensar". Que la palabra "es", quiere decir: "La palabra forma parte de la irradiación del esenciar del ser, cumpliendo así una fidelidad suprema con su esencia única" ${ }^{58}$. Esta es la fidelidad fenomenológica al acontecimiento del ser. Por su parte, la "meditación" es el esfuerzo pensante por captar las señas esenciales que se dan como palabra,

La meditación traslada al hombre al Da-sein, supuesto que ella misma sea ya acaecida-apropiadoramente por el ser (Seyn). Pero el ser (Seyn) reclama a la palabra, como la que el evento-apropiador respectivamente se esencia ${ }^{59}$.

De modo que en tanto meditación se da la "fundación del Dasein", en el "ensamble simultaneo" de Ereignis, meditación y palabra fundamental. Pensar es estar situado ya en lo abierto del Dasein, pensar es ya fundar el Dasein, pero toda vez que ello está aún puesto en decisión, tendremos que admitir que aún no podemos o no sabemos todavía pensar, como afirmará Heidegger en los años ‘ $50^{60}$.

De acuerdo con esto se entiende por qué desparece el término fenomenología, en favor del sobrio "pensar".

La palabra pensar (Er-denken) quiere decir: el pensar que es antes acaecidoapropiadoramente por el ser (Seyn), lo por pensar, y es realizable sólo en una historia y como historia del ser (Seyn) ${ }^{61}$.

La fenomenología en tanto método, es aún un emprendimiento que parte del ser humano, pero dado que su objetivo temático, la verdad del ser, es lo que precisamente se rehúsa a todo emprendimiento humano, la metodología debe saber sustraerse a la voluntad planificadora. Por eso pregunta el filósofo:

¿Pero pensamos "nosotros" al ser (Seyn)? ¿O "es" el ser (Seyn), en tanto acaece-apropiadoramente al pensar (no un representar cualquiera sino el pensar (Erdenken) de él) y con ello a un esenciarse del hombre?62

Por esta razón, las señas (Winke), como apuesta nominativa de una fenomenología inaparente, no son solo palabras "del" filósofo, que surgen de su sensibilidad, inteligencia

58 Ib. p. 244.

59 HEIDEGGER, M. Meditación. cit. p. 56.

60 Cfr. HEIDEGGER, M. ¿Qué significa pensar? Madrid, Trotta, 2010.

61 HEIDEGGER, M. Meditación. cit. p. 303.

62 lb. p. 118. 
o astucia, sino indicio de que el pensador logra escuchar, captar las propias señas que el ser deja en su retirada. De este modo, el Seynsdenken “(...) no saca a rastras un significado de las palabras abordadas para ofrecerlas como si ellas fueran la 'cosa misma', sino que le toma la palabra al propio ser (Seyn)"63. Si la esencia de la verdad del ser es "rehusante", la palabra esencial también es rehúso, por ello nuestra experiencia del lenguaje para con el ser resulta siempre con la apariencia de "insuficiente y oscura".

A partir de lo dicho, resulta notorio que en esta nueva comprensión de la fenomenología el propio lenguaje de los existenciarios resulta insuficiente.

Aunque los "existenciarios" están referidos al Da-sein, y por tanto están referidos exclusivamente a la pregunta por la verdad del ser, no se los piensa lo bastante primordialmente desde la irradiación del esenciar del ser ${ }^{64}$.

Por ello dan la impresión de constituir, a pesar de las constantes advertencias del autor, "categorías" del ser humano. Esto no significa, como se ha apuntado previamente, que los existenciarios deban desaparecer, sino que deben ser replanteados y renombrados de acuerdo con la perspectiva de una fenomenología inaparente. Un trabajo pendiente sería ahondar en el modo como estas estructuras existenciarias son replanteadas y renombradas de acuerdo con las "señas" del pensamiento ontohistórico.

\section{Bibliografía}

BASSO, L. La unidad de la diferencia. Acerca del acontecer en la obra de Heidegger. Buenos Aires. Biblos, 2017.

cosmus, O. Anonyme Phänomenologie. Die Einheit von Heideggers Denkweg. Würzburg, Königshausen \& Neuman, 2001.

GADAMER, H.-G. Los caminos de Heidegger. Barcelona, Herder, 2002.

GethmANN, C. Verstehen und Auslegung. Das Methodenproblem in der Philosophie Martin Heideggers. Bonn, Bouvier, 1974.

HEIDEgGeR, M. GA 66. Besinnung. Fráncfort del Meno, Vittorio Klostermann, 1997.

HEIDEGGER, M. Los problemas fundamentales de la fenomenología. Madrid, Trotta, 2000. HEIDEGGER, M. Meditación. Buenos Aires, Biblos, 2006.

63 HEIDEGGER, M. Cuadernos negros (1938-1939). cit. p. 260.

64 lb. p. 261 (Trad. modificada). 
HEIDEGGER, M. Sobre el comienzo. Buenos Aires, Biblos, 2007.

HEIDEGger, M. Hitos. Madrid, Alianza, 2007.

Heidegger, M. Preguntas fundamentales de la filosofía. "Problemas" selectos de "lógica". Granada, Comares, 2008.

HEIDEGGER, M.: ¿Qué significa pensar? Madrid, Trotta, 2010.

HEIDEGGER, M.: Tiempo y ser. Madrid, Tecnos, 2011.

HEIDEGGER, M.: Ser y tiempo. Madrid, Trotta, 2012.

HEIDEGGER, M.: Cuadernos negros (1931-1938). Madrid, Trotta, 2015

HEIDEGGER, M.: Cuadernos negros (1938-1939). Madrid, Trotta, 2017.

HEIDEGGER, M. GA 82. Zu eigenen Veröffentlichungen. Fráncfort del Meno, Vittorio Klostermann, 2018.

HERRMANN, F.-W. von. Weg und Methode. Zur hermeneutischen Phänomenologie des seinsgeschichtlichen Denkens. Fráncfort del Meno, Vittorio Klostermann, 1990.

HERRMANN, F.-W. von. La segunda mitad de Ser y tiempo. Sobre Los Problemas fundamentales de la fenomenología de Heidegger. Madrid, Trotta, 1997.

HUSSERL, E. Investigaciones lógicas I. Madrid, Alianza, 1999.

HUSSERL, E. Ideas relativas a una fenomenología pura y una filosofía fenomenológica. Libro primero: Introducción general a la fenomenología pura (Ideas I). México D.F., UNAM/FCE, 2013.

PÖGgELER, O. El camino del pensar de Martin Heidegger. Madrid, Alianza, 1993.

POLT, R.: "The Black Notebooks as Thought Journals", en DENKER, A. y ZABOROWSKI, H. (eds.), Heidegger-Jahrbuch 11. Zur Hermeneutik der "Schwarzen hefte". Freiburg/Múnich, Karl Alber, 2017.

SAfRANSKI, R. Un maestro de Alemania. Martin Heidegger y su tiempo. México D.F., Tusquets, 2010.

THOMSON, I.: “Heidegger's Nazism in the Light of his early Black Notebooks: A view from America", en DENKER, A. y ZABOROWSKI, H. (eds.), Heidegger-Jahrbuch 11. Zur Hermeneutik der "Schwarzen hefte". Freiburg/Münich: Karl Alber, 2017. 
VAllegA-NEU, D. "Die Schwarzen Hefte und Heideggers seynsgeschichtliche Abhandlungen (1936-1942)“, en DENKER, A. y ZABOROWSKI, H. (eds.). Heidegger-Jahrbuch 11. Zur Hermeneutik der "Schwarzen hefte". Freiburg/Múnich, Karl Alber, 2017.

VIGo, A. Arqueología y aleteiología y otros estudios heideggerianos. Buenos Aires, Biblos, 2008.

VOLPI, F. "Goodbye, Heidegger! Mi Introducción Censurada a los Beiträge zur Philosophie", en Actas del I Congreso Internacional de Fenomenología y Hermenéutica. Santiago de Chile, Universidad Andrés Bello, 2008.

VOLPI, F. Martin Heidegger. Aportes a la filosofía. Madrid, Maia, 2010.

xolocotzl, A. Fenomenología de la vida fáctica. Heidegger y su camino a Ser y tiempo. México D.F., UIA/Plaza y Valdés, 2004.

xolocotzI, A. Fundamento y abismo. Aproximaciones al Heidegger tardío. México D.F., BUAP/Porrúa, 2011.

xolocotzl, A. y Zırıón, A. iA las cosas mismas! Dos ideas sobre fenomenología. Ciudad de México, Porrúa/Universidad Michoacana de San Nicolás Hidalgo/BUAP, 2018. 